the trance personality, has a vacabulary limited to the few words used by 'her' during the ordinary sittings.

It has been alleged that the trance personality is aware of what goes on in the dark séance-room ${ }^{2,8}$; in these sittings this was not found to be the case.

The rate of breathing of the medium in trance ranged, in thess sittings, from 90 to 260 eycles per minute, continuing with two or three intervals of some 15 minutes each, for 5 to $6 \frac{1}{2}$ hours. The longest continuous period was $2 \frac{1}{2}$ hours, with frequent stops totalling about 10 minutes. In view of the fact that this respiration has been regarded as a remarkable physiological phenomenon, samples were collected and analysed by Dr. C. G. Douglas, showing that the medium in no way overbreathes. As the rate of respiration increases so its depth decreases; the total oxygen consumption per minute observed never exceeded 1410 c.c. at N.T.P., which corresponds to a man walking some four miles an hour. During trance this medium is in constant, often violent movement, so that these results are in no way paranormal. Moreover, we have found no difficulty in imitating his breathing.

Every suggestion made by the medium and the trance personality was acted upon, and both repeatedly expressed their satisfaction with the arrangements and with the investigators. On our side it is impossible to speak too highly of Rudi Schneider's willingness to submit to every suggested test and control; he acted throughout with the most scrupulous straightforwardness.

TheOdORE Besterman. OLIVEr Gatry.

Society for Psychical Research, 31 Tavistock Square, W.C.1.

${ }^{1}$ NATURE, 182, 776, 801, 849; 1933.

2 E. and M. Osty, "Les pouvoirs inconnus de l'esprit sur la matière", 1932.

C. V. C. Herbert, Proc. S.P.R., 41, 259 ; 1933.

4 Lord Rayleigh, Proc. S.P.R., 41, 89, $269 ; 1933$

5 E. Osty, "Supernormal Aspects of Energy and Matter" (F. W. H. Myers Memorial Lecture), pp. 18, 30; 1933 .

H. Price, "Rudi Schneider" 1930;" "An Account of some further Experiments with Rudi Schneider", 1933.

\section{Transformation of Yellow Mercuric Iodide into the Red Form}

RoDwell and Elder ${ }^{1}$ have observed microscopically that when yellow orthorhombic crystals of mercuric iodide are touched, the change into the red variety continues through the whole mass of crystals, and the resulting pseudomorph consists of minute octahedrons of the red form.

It is well known that the yellow form crystallises from a solution of mercuric iodide in alcohol, and exists for varying lengths of time. On viewing under the microscope single crystals free from etched markings, the change from the yellow into the red variety is found to be in accordance with the usual type observed in solid reactions. After about 15 minutes, nucleation usually occurs along the whole length of the crystal edge; the interface then advanees rapidly across the crystal parallel to the opposite edge. Occasionally nucleation occurs as a thin strip in the interior of the crystal parallel to the edges; the interface then moves out towards the edges of the crystal with approximately the same linear rate in both directions. It appears that nucleation occurs along some weak axis in the crystal, and immediately spreads along the whole length; the reaction proceeds with the usual parallel advance. At $20^{\circ} \mathrm{C}$. the mean linear rate of advance of the interface was $0.0025 \mathrm{~cm}$. $/ \mathrm{sec}$

By heating the red variety, very much smaller rhombohedra of the yellow form were volatilised on to a cover-glass. Such crystals commenced to change into the red form after about five hours, the reaction spreading inwards very slowly from all four odges with the usual parallel advance of the interface; octahedra of the red form could be observed in the decomposed part of the crystal.

The linear advance of the interface parallel to the edges of the crystal has previously been observed in the case of true decomposition, for example, potassium hydrogen oxalate hemihydrate ${ }^{2}$ and potassium chlorate $^{3}$, and it appears to be characteristic also of the transition from one crystalline form into another of polymorphic substances.

Investigation of the reaction is proceeding.

Robert Gordon's Colleges, JoHN B. M. COPPOCK. Aberdeen.

1 Rodwell and Elder, Proc Roy. Soc., 28, 284; 1879.

2 Hume and Colvin, Proc, Roy Soc, A, 125, 635; 1929.

${ }^{3}$ Coppock, Colvin and Hume, Trans. Far. Soc., 27, 283 ; 1931.

\section{Rate of Nucleation of Copper Sulphate in Vacuum}

THE rate of growth of the centres of dehydration of $\mathrm{CuSO}_{4} .5 \mathrm{H}_{2} \mathrm{O}$ varies with the crystal direction. On the large faces of the crystal, the nuclei grow in the form of a cross and the direction of the arms of the cross are parallel to two of the crystal axes. The underlying surfaces of the nuclei prove to be very complex, for a disc of dehydrated material is found to be suspended from the longer arm of the cross. This dise passes into the crystal at an angle of approximately $\mathbf{3 3}^{\circ}$.

Counts have been made of the rate of production of centres of decomposition on the surfaces of a crystal, when this has been removed from the saturated solution, carefully dried and placed in a high vacuum. No visible nuclei appear at room temperature until after an induction period which is of the order of 100 minutes at $18^{\circ} \mathrm{C}$. Thereafter, the number of nuclei increases at a linear rate. The length of the induction period decreases with increasing temperature, but on account of variations in the behaviour of individual crystals it has not been possible to determine the temperature coefficient. Scratched crystals give shorter induction periods, and crystals with irregular surfaces give larger numbers of centres than more perfect crystals.

The induction period is most probably due to the slow rate of growth of the nuclei when first formed, this rate being much slower than that of visible nuclei. It is thus incorrect to assume in all cases of solid decomposition that the outward rate of growth of the nuclei is constant at all stages in their growth'. In the decomposition of barium azide where the rate of decomposition is given by $d p / d t \sim t^{10}$ and in that of mercury fulminate where $d p / d t \sim t^{\mathbf{2 4}}$, the observed induction periods are in the main to be ascribed to an accelerating rate of nuclear growth. N. F. H. BRIGHT.

The University, W. E. Garner.

Bristol.

March 16.

${ }^{1}$ Cf. S. V. Izmailov, Phys. Z. Sowjet Union, 4, $835 ; 1934$. 